\title{
Cost Modeling of Backhaul for Mobile Networks
}

\author{
Mozhgan Mahloo, Paolo Monti, Jiajia Chen and Lena Wosinska \\ KTH Royal Institute of Technology, School of Information and Communication Technology, \\ Isafjordsgatan 22, Electrum 229, 16440 Kista, Sweden \\ Email: \{mahloo, pmonti, jiajiac,wosinska\}@kth.se
}

\begin{abstract}
Wireless heterogeneous networks (HetNets) based on small cells are a cost and energy efficient alternative to provide high capacity to the end users. On the other hand, the cost and energy consumption of backhaul network aggregating data traffic from a large number of base stations may limit the benefits brought by the use of small cell. In HetNet deployments it becomes thus essential to be able to assess the total cost of ownership (TCO) of the backhaul network. This paper presents for the first time a comprehensive cost evaluation methodology to compute the TCO of mobile backhaul networks. The presented model can be instrumental to identify the most critical cost drivers in the backhaul networks and to have a better understanding of the backhaul TCO dynamics when small cells are deployed. The proposed TCO model is then used in a case study where two technology options for the backhaul are considered, i.e., microwave and fiber. The results from the case study show how it is possible to identify the most critical cost factors, thus easing the way towards a cost efficient backhaul design strategy.
\end{abstract}

Index Terms-Backhaul, total cost of ownership (TCO), cost modeling, fiber, microwave, heterogeneous wireless networks.

\section{INTRODUCTION}

In the recent years the exponential growth of mobile traffic, mainly driven by an increase in the demand for video services and number of mobile devices, brings new challenges for mobile network operators (MNOs). Traditionally this capacity growth is addressed by acquiring new spectrum, enhancing spectrum efficiency, and/or adding new macro cell sites. However, the spectrum is not an infinite resource and spectrum efficiency is improving at a much lower rate than the capacity demand is increasing. In addition, in dense urban areas there are difficulties to acquire new base station (BS) sites and to serve a growing number of users that mainly reside indoor. A promising way to solve the current (and future) capacity crunch is to deploy wireless heterogeneous networks (HetNets), where high power macro cells provide coverage, and small outdoor/indoor cells are deployed close to the end user to ensure that capacity is provided only where is needed in a cost and energy efficient way.

The benefits of HetNets over homogeneous deployments (i.e., with macro cells only) have been demonstrated in a number of studies in terms of both cost and power consumption [1][2]. For example, the study in [2] emphasized how a HetNet deployment is a cost efficient alternative especially in scenarios where the capacity demands is higher than $100 \mathrm{Mbps} / \mathrm{km}^{2}$.
On the other hand the introduction of small cells has an impact on the part of the network referred to as backhaul, which is responsible for collecting data traffic from the BSs and for sending it to the metro/aggregation network mostly in terms of number of required backhaul connections and their capacity. In fact, a large number of links is required to aggregate the data traffic originating at each small cell, each one working at a peak rate of tens of Mbps. These rates cannot be guaranteed with legacy copper-based infrastructures (i.e., at least not over long distances) thus forcing mobile operators to upgrade their backhaul networks to avoid potential bottlenecks, with obvious consequences in terms of additional costs. Since the backhaul cost is already a not negligible part of the total cost of ownership (TCO) in homogeneous wireless networks[3], the impact of the mobile backhaul segment on the TCO will become even more crucial with an increasing number of small cells used in future HetNet deployment [4]. Therefore, it is important that current and future mobile HetNet deployments are designed considering cost efficient backhaul architecture. This can be done only with the help of a detailed TCO modeling to evaluate the various cost factors (covering deployment and operational processes) for the different types of backhaul networks. Otherwise, the backhaul cost might limit the benefits brought by small cell deployments.

There are some attempts in the literature to provide such modeling. The works in [5][6] study various wireless architectures (both homogeneous and HetNet) and try to assess the impact of backhaul on the entire TCO. However, these studies do not consider different types of backhaul architectures and technologies and their benefits in terms of cost saving. In [7][8] different microwave backhaul topologies (including mesh and tree) are compared with respect to their total cost. It was concluded that mesh structures are an efficient option considering homogeneous wireless deployment. The study in [9] compares the deployment cost of backhauling a long term evolution (LTE) wireless network using fiber, microwave and E-band technologies. It is shown that for scenarios with low cell density, microwave is the cheapest option. However, only a homogeneous wireless deployment was evaluated in this work. The work in [10] compares different technology and topology options for backhauling a HetNet deployment, but only in terms of energy, while a more general TCO assessment is not provided. To the best of our knowledge a complete assessment (i.e., considering the entire TCO) of the impact of a given backhaul technology on a HetNet deployment is not available so far. 
In this paper we derive a comprehensive cost model with a detailed capital expenditure (CAPEX) and operational expenditure (OPEX) breakdown. The proposed methodology is general enough to be adapted to different backhaul technologies and architectures and it allows identifying the most significant cost factors in the backhaul segment and consequently to improving the overall network cost efficiency. Furthermore, we use this model to perform a case study to assess the TCO of backhaul deployment in a dense urban area where two technological options for the backhaul are considered (i.e., a microwave- and a fiber-based architecture). The results from the case study highlight the most critical TCO aspects for the two technologies under exam.

\section{MoBile BACKHAUL TECHNOLOGICAL AND ARCHITECTURAL OPTIONS}

A backhaul network is responsible for aggregating and sending data traffic from the radio access to the (wired) backbone segment. Operators usually select a suitable backhaul technology (or a combination of them) according to their needs in terms of capacity, reliability, cost, and deployment time. From the technological point of view backhaul network can be based on copper, fiber, and microwave [11]. Microwave represents nearly $50 \%$ of all current backhaul deployments and it is expected that it will maintain the same share in the years to come. This is mainly due to its moderate installation cost and relatively short time to deploy. Copper-based backhaul segments amount approximately to $20 \%$ of all current deployments. Most likely, they will be gradually replaced due to the their limited capacity over long distances (i.e., more than hundred Mbps can be guaranteed only up to 500 meters) and their inability to scale in a cost efficient manner with the number of BSs. Fiber-based backhaul, on the other hand, offers virtually unlimited capacity over long distances. However, it is relatively expensive and slow to deploy in areas where no fiber infrastructure already exists. However, a widespread penetration of fiber, mostly replacing existing copper-based infrastructures, can be expected in the coming years, since fiber has been recognized as the future-proof technology for broadband access. Therefore, it can be assumed that fiber and microwave will be the two main candidates for backhauling of the future mobile networks, and for this reason we focus our analysis only on these two technologies.

In terms of possible architectures, regardless of the specific topology in which they are organized, a microwave-based backhaul consists of a number of point-to-point (PtP) microwave links, each one requiring two antennas. One antenna is connected to the BS whose data traffic needs to be backhauled, while the other antenna is connected either to a switch at the first aggregation point of the backhaul infrastructure (i.e., in the case of a multistage backhaul) or directly to a switch at the metro node. When several microwave antennas are co-located in one place a tower mast needs to be installed which is referred to as the microwave hub.

Fiber backhaul networks are organized either in tree-based or in PtP topologies. In the case of PtP, one optical line terminal (OLT) located in a central office $(\mathrm{CO})$ is connected to an optical network unit (ONU) at the user promises via a dedicated fiber. With tree-based architecture, e.g., passive optical network (PON) or active optical network (AON), each
OLT is connected to several ONUs via a splitting/switching device in the cabinet. More detailed description of possible backhaul architectures can be found in [10].

\section{Cost Modeling}

This section presents a comprehensive TCO model covering both CAPEX and OPEX aspects of a backhaul segment. More specifically, the model includes all the costs incurred during the backhaul life cycle (i.e., from the network deployment phase, when a huge upfront investment is required, up to all cost aspects related to each operational process).

Fig. 1 presents a cost classification according to the proposed cost model for the mobile backhaul network. Since, in general, a backhaul segment can comprise more than one technology (i.e., a hybrid architecture), the proposed model accounts for the presence of both fiber and microwave. The details of each part are presented next.

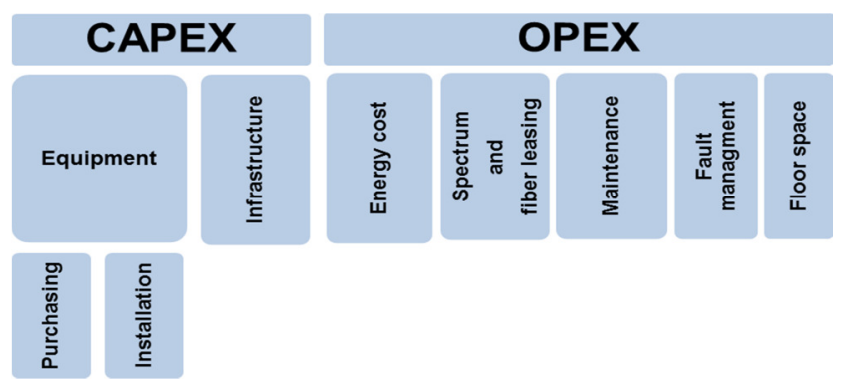

Fig. 1. Cost classification of TCO model of backhaul.

\section{A. CAPEX}

The CAPEX refers to all the expenses related to having the backhaul network in place. According to the proposed model (Fig.1), CAPEX can be divided into two main parts, i.e., equipment and infrastructure cost. They are described next.

\section{Equipment cost}

The Equipment cost ( $\operatorname{Tot}_{E q}^{\text {cost }}$ ) is the sum of all expenses related to purchasing the backhaul components $\left(E q_{\text {Pur }}\right)$ according to the results of the network dimensioning phase and to install them in their specific locations $\left(E q_{\text {Inst }}\right)$ :

$$
T o t_{E q}^{\cos t}=E q_{\text {Pur }}+E q_{\text {Inst }}
$$

The purchasing cost $\left(E q_{\text {Pur }}\right)$ can be defined as:

$$
E q_{\text {Pur }}=E q_{\text {Pur }}^{F}+E q_{\text {Pur }}^{M w}
$$

$E q_{\text {Pur }}^{F}$ represents the total cost for purchasing the fiber network components which can be computed by Equation 3:

$$
E q_{\text {Pur }}^{F}=\sum_{i=1}^{N_{t}} V_{i}^{e q} P r_{i}^{e q},
$$

where $N_{t}$ denotes the number of component types (e.g., OLTs, ONUs, splitters). $V_{i}^{e q}$ and $\operatorname{Pr}_{i}^{e q}$ represent the volume (i.e., the number of units) and the price of equipment $i$, respectively. Both the number of equipment types and their quantity may vary depending on the specific architecture chosen for the fiber backhaul part (e.g., PtP vs. tree-based, active vs. passive). The purchasing cost of the microwave equipment can be obtained as follows: 


$$
E q_{P u r}^{M w}=2 N_{M W l i n k} P r_{a n t}+N_{s w} P r_{s w},
$$

where $N_{\text {MWlink }}$ and $P r_{\text {ant }}$ represent the total number of microwave links in the backhaul segment, and the price of each antenna, respectively. $N_{s w}$ and $P r_{s w}$ denote the number of aggregation switches and the price of each switch connected to microwave antennas. Once the backhaul equipment is purchased a technician has to spend a certain amount of time to install and test each component. This cost can be expressed as follows:

$$
E q_{\text {Inst }}=E q_{\text {Inst }}^{F}+E q_{\text {Inst }}^{M w}
$$

The total installation cost is divided into fiber installation ( $\left.E q_{\text {Inst }}^{F}\right)$ and microwave installation $\left(E q_{\text {Inst }}^{M w}\right)$ cost. They are computed using Equation 6 and Equation 7, respectively.

$$
E q_{\text {Inst }}^{F}=\left[\sum_{j=1}^{N_{\text {loc }}}\left(\left(\sum_{i=1}^{N_{t}} T_{i j}^{E q I n} V_{i j}^{E q}\right)+2 N_{j}^{\text {Tech }} T_{j}^{\text {tra }}\right)+2 N_{F i b} T_{\text {Spl }}\right] T e c h_{\text {sal }}
$$

$N_{l o c}$ denote the number of nodes where the backhaul equipment is placed. $T_{i j}^{E q I n}$ and $V_{i j}^{E q}$ in Equation 6 represent the installation time of equipment $i$ and its volume in location $\mathrm{j}$, respectively. The travelling time of a technician to/from location $j$ and the number of technicians sent to that location are denoted by $T_{j}^{t r a}$ and $N_{j}^{T e c h} . N_{F i b}$ represents the total number of connected fibers used in the backhaul segment while $T_{S p l}$ is the time required to splice and connect each fiber. The total installation time is multiplied by the hourly technician rate $\left(T e c h_{\text {sal }}\right)$ to obtain the total installation cost.

$$
E q_{\text {Inst }}^{M w}=\left[\sum_{i=1}^{N_{M W l i n k}}\left(T_{i}^{\text {antIn }}+2 T_{i}^{\text {tra }}\right) N_{t} N_{\text {team }}^{\text {tech }}+T_{\text {SwIn }} N_{S w}\right] \text { Tech }{ }_{\text {sal }}
$$

$N_{M W l i n k}, N_{t}$ and $N_{\text {team }}^{\text {tech }}$ denote the number of microwave links, the number of teams (i.e., two in case of a PtP microwave link to ensure the correct antenna alignment) needed per each link, and the number of technicians per team. The time to install a microwave antenna and the traveling time to the location of each antenna are represented by $T_{i}^{a n t I n}$ and $T_{i}^{\text {tra }}$, respectively. The installation time for the switches connected to the microwave links is calculated by multiplying the installation time of a switch $\left(T_{S w I n}\right)$ by total number of switches $\left(N_{S w}\right)$ used in the backhaul network.

\section{Infrastructure cost}

The total infrastructure cost ( $\operatorname{Tot}_{\operatorname{lnf} f a}^{\cos t}$ ) of a mobile backhaul segment corresponds to the investment needed to deploy the fiber infrastructure $\left(F i b_{\text {inf }}\right)$ as well as cost of leasing fibers (when the fiber infrastructure has already been deployed by other providers and is available for leasing). Finally $\operatorname{Tot}_{\text {Infra }}^{{ }^{\cos t}}$ also includes the expenses needed to install the microwave hubs, i.e., masts and antennas, where needed.

$$
\operatorname{Tot}_{\text {lnfra }}^{\text {cost }}=F i b_{\text {inf }}+\sum_{i=1}^{N_{\text {lease }}} L_{i} \operatorname{Pr}_{\text {leasing }}+N_{\text {Mwhb }} \operatorname{Pr}_{\text {hub }}^{\text {install }}
$$

The optical fibers are placed inside the ducts that are buried under the ground (trenching). The fiber infrastructure cost includes all the expenses related to trenching, purchasing of fiber cables, and pumping fibers into the ducts. This expense can be expressed as follows:

$$
F i b_{\text {inf }}=L_{\text {trench }} P r_{\text {trench }}+L_{\text {fib }} \operatorname{Pr}_{\text {fib }}+L_{\text {pump }} P r_{\text {pump }},
$$

where $L_{\text {trench }}, P r_{\text {trench }}, L_{f i b}$, and $P r_{f i b}$ denote total trenching length, the price of trenching per kilometer $(\mathrm{km})$, the amount of fiber (i.e., total length in $\mathrm{km}$ ) to be purchased, and the price of one $\mathrm{km}$ of fiber, respectively. When empty ducts are available, a new fiber can be pumped into them, hence no trenching is required in these areas. This is reflected by the $L_{\text {pump }}$ parameter, i.e., length of the fiber to be installed in the existing ducts, and the cost per $\mathrm{km}$ for pumping the fiber $\left(P r_{\text {pump }}\right)$.

$N_{M W h u b}$ and $\operatorname{Pr}_{\text {hub }}^{\text {install }}$ in (8), represent the number of microwave hubs and their installation cost. In many cases, MNOs prefer to lease fibers instead of deploying their own infrastructure. In such cases, the infrastructure cost includes an upfront charge per $\mathrm{km}$ of leased fiber paid to the infrastructure owner $\left(P r_{\text {leasing }}\right)$. The number of leased connection and their length are given by $N_{\text {lease }}$ and $L_{i}$, respectively.

\section{B. OPEX}

OPEX refers to the expenses occurred during network operation over a predefined time interval (i.e., the network operational time). The main OPEX components are indicated in Fig. 1 and they are defined below. The following equations present the yearly OPEX, which needs to be multiplied by the network operational time to calculate the total OPEX.

\section{Spectrum and fiber leasing}

The spectrum and fiber leasing cost can be expressed by Equation 10:

$$
\operatorname{Tot}_{\text {Leas }}^{\mathrm{cos} t}=\sum_{i=1}^{\mathrm{N}_{\mathrm{cc}}}\left(N_{c_{i}}^{M W \text { ink }} \operatorname{Pr}_{c_{i}}^{\text {MWink }}\right)+L_{\text {Lease }} \operatorname{Pr}_{\text {Leas }}^{\text {year }}
$$

When leasing fibers, a MNO is charged a yearly fee for the maintenance and reparation of the rented fibers in addition to the upfront expenses. This cost is computed by multiplying the total length of leased fibers in kilometer $\left(L_{\text {lease }}\right)$ by the yearly maintenance fee per kilometer $\left(P r_{\text {Leas }}^{\text {year }}\right) . N_{c c}$ and $N_{c_{i}}^{M W l i n k}$ denote the number of capacity classes of the microwave links used for the backhaul (e.g., $100 \mathrm{Mbps}, 500 \mathrm{Mbps}$ ), and the number of microwave links belonging to capacity class $i$, respectively. The yearly cost of spectrum leasing for a licensed microwave link is denoted by $\operatorname{Pr}_{c_{i}}^{\text {MWlink }}$. This quantity varies depending on channel capacity (i.e., class $i$ ) and the frequency band.

\section{Energy cost}

The electricity cost ( $\operatorname{Tot}_{\text {Energy }}^{\cos t}$ ) is obtained by summing up the energy cost of all the active equipment in the various backhaul locations (i.e., CO, cabinets, microwave sites).

$$
\operatorname{Tot}_{\text {Energy }}^{\cos t}=C o_{E n}^{\cos t}+C a b_{E n}^{\cos t}+M W_{E n}^{\cos t}+I n_{E n}^{\cos t}
$$

The CO energy cost ( $C o_{E n}^{\cos t}$ ) can be expressed as: 


$$
C o_{E n}^{\text {cost }}=N_{c o} \operatorname{Co}_{f i x / y}+a_{1} \sum_{i=1}^{N_{c o}} \operatorname{Co}_{i}^{E n} \operatorname{Pr}_{K w h}^{\text {indoor }}
$$

where $N_{c o}$ and $C o_{f i x y}$ denote, respectively, the number of central offices, and the fixed yearly connection fee paid by the MNO to the electricity supplier based on the predicted energy consumption. The variable " $a_{l}$ " is a coefficient used to account for the energy consumed for the cooling and the housing of the equipment. $P r_{K w h}^{\text {indoor }}$ and $C o_{i}^{E n}$ represent the price per energy unit (i.e., $\mathrm{kWh}$ ) for the indoor equipment, and the total energy consumption of all components inside the central office $i$, respectively. The energy cost of cabinet locations $\left(C a b_{E n}^{\cos t}\right)$ is given by the following equation.

$$
C a b_{E n}^{\cos t}=a_{2} \sum_{i=1}^{N_{c a b}} \operatorname{Cab}_{i}^{\text {En }} \operatorname{Pr}_{K w h}^{\text {outdoor }}
$$

$N_{c a b}, C a b_{i}^{E n}$ and $\operatorname{Pr}_{K w h}^{\text {outdoor }}$ denote the number of cabinets, the total energy consumption of the equipment at cabinet $i$, and the price per unit of energy for outdoor equipment, respectively. The " $a_{2}$ " is the cooling coefficient for cabinets.

The energy consumption of microwave links is calculated as follows:

$$
M W_{E n}^{\cos t}=a_{3} \sum_{i=1}^{N_{M W \text { site }}} M W_{i}^{E n} \operatorname{Pr}_{K w h}^{\text {outdoor }}
$$

where $N_{M W \text { site }}, M W_{i}^{E n}$, and $\mathrm{a}_{3}$ represent the number of microwave sites where antennas are located, the energy consumed in each site and the cooling coefficient of each microwave site, respectively.

The equipment located in the buildings connected to the indoor cells, e.g., ONUs, also consume power. This fee is normally paid by the users and is defined as follow:

$$
\operatorname{In}_{E n}^{\mathrm{cos} t}=\sum_{i=1}^{N_{B l}} \text { Inhouse }_{i}^{\text {En }} \operatorname{Pr}_{K w h}^{\text {user }}
$$

where $N_{B l}$, Inhouse ${ }_{i}^{E n}$ and $\operatorname{Pr}_{K w h}^{\text {user }}$ represent the number of buildings with indoor cells, the energy consumption of backhaul equipment per building and the price per unit of energy for residential customers, respectively.

\section{Maintenance cost}

A regular maintenance routine is needed to keep a backhaul network up and running. This includes monitoring and testing the equipment, updating the software (including renewing licenses when needed), and the renewal of supporting components such as batteries, etc. The total maintenance cost ( $\operatorname{Tot}_{M}^{\cos t}$ ) is expressed by Equation 16:

$$
\operatorname{Tot}_{M}^{\mathrm{cos} t}=C o_{M}+C a b_{M}+M W_{M}+S W_{\text {lic }},
$$

where $C o_{M}, C a b_{M}$ and $M W_{M}$ reflect the maintenance cost of central offices, cabinets and microwave links, respectively. The yearly fee paid for the software licenses are represented by $S W_{\text {lic }}$. Operators consider several rounds of maintenance procedures for each central office depending on the number of users and services covered by each one of them. This expense can be modeled as follows:

$$
C o_{M}=N_{C o}^{M h} N_{C o} \text { Tech }_{\text {sal }}+M_{C o}^{\cos t} N_{C o},
$$

where $N_{C o}^{M h}, N_{C o}$ and $M_{C o}^{\cos t}$ denote the man-hours required for the maintenance of each central office per year, the number of central offices and the fixed cost to be paid for hardware upgrade, and for replacing some materials (e.g., batteries), respectively.

A similar expression can be derived for the maintenance of cabinets (Equation 18), where the number of man-hours per cabinet ( $N_{c a b}^{M h}$ ) is lower compared to one used for the central offices.

$$
C a b_{M}=\sum_{i=1}^{N_{c a b}}\left(N_{c a b}^{M h}+2 T_{c a b_{i}}^{\text {trav }}\right) T e c h_{s a l}+M_{c a b}^{\text {cost }} N_{c a b}
$$

$N_{c a b}$ represents the number of cabinets and $M_{\mathrm{c} a b}^{\mathrm{cos} t}$ denotes the cost to be paid per year for hardware upgrade and for replacing some components that are worn out (e.g., batteries).

Microwave links also require a regular monitoring, because antennas might tilt and lose their line of sight. This part of the TCO can be expressed by Equation 19.

$$
M W_{M}=\sum_{i=1}^{N_{M W a n t}}\left(N_{M W a n t}^{M h}+2 T_{\text {MWant }_{i}}^{\text {trav }}\right) \operatorname{Tech}_{\text {sal }}
$$

Here $N_{M \text { Want }}^{M h}$ and $T_{M \text { Want }_{i}}^{\text {trav }}$ represent yearly man-hours required for the maintenance of each microwave antenna and the travelling time to the location of each antenna, respectively.

\section{Fault management}

Fault management refers to the expenses related to the reparation of the failures that might occur in a backhaul network. The average number of failures per year of each component type $i\left(A N F_{i}\right)$ can be calculated based on the component failure rate. $A N F_{i}$ multiplied by the number of equipment of type $i$ ( $N_{i}^{e q}$ ) in the backhaul network gives the expected number of failed components of type $i$ during one year. Equation 20 defines the average total yearly reparation cost for the backhaul network as the sum of the reparation cost of each failure occurring during the year.

$$
T o t_{F M}^{\mathrm{cos} t}=\sum_{i=1}^{\substack{\text { ceq } \\ \text { type }}}\left(\left(M T T R_{i}+2 T_{\text {trav }}\right) N_{\text {tech }} T e c h_{\text {sal }}+E q_{i}^{\text {cost }}\right) A N F_{i} \times N_{i}^{e q}
$$

The reparation cost depends on the cost of purchasing new component when needed $\left(E q_{i}^{\text {cost }}\right)$, mean time to repair (MTTR) of each device/network segment $i$, and the travelling time to the location of the failure $\left(T_{\text {trav }}\right) . N_{\text {type }}^{e q}$ and $N_{\text {tech }}$ denote the number of equipment types and the number of technicians required to repair a failure, respectively.

\section{Floor space cost}

The floor space cost $\left(T o t_{F I S p}^{\cos t}\right)$ is a yearly rental fee paid by an operator to house its equipment, i.e., to place components in 
the racks with standard size in various locations. $A_{R}$ defines the space required for a rack including enough working space in front of it for the technicians to operate on the rack. The number of racks inside a central office is computed by dividing the number of equipment per central office $\left(N_{c o}^{E q}\right)$ by the number of equipment per rack $\left(N_{\text {Rack }}^{E q}\right)$. The total floor space cost can be defined as follows:

$$
\operatorname{Tot}_{F l s p}^{\text {cost }}=\left(A_{R} \frac{N_{c o}^{E q}}{N_{R a c k}^{E q}}\right) \operatorname{Pr}_{m^{2}}^{I n}+\left(A_{C} N_{C}+A_{h u b} N_{h u b}+A_{M W} N_{M W}\right) \operatorname{Pr}_{m^{2}}^{\text {Out }} \text {, }
$$

where $\operatorname{Pr}_{m^{2}}^{\text {In }}$ and $\operatorname{Pr}_{m^{2}}^{\text {Out }}$ are, respectively, the yearly rental fee paid by an operator for indoor areas (e.g., CO) and outdoor locations where no housing is provided. Cabinets are usually built with a standard size $\left(A_{C}\right)$ regardless of amount of components inside them. $N_{C}$ and $N_{h u b}$ represent the number of cabinets and hubs in the backhaul, while $A_{h u b}$ shows the area required to install a microwave hub.

\section{CASE STUDY}

This section presents a case study where the proposed TCO model is used to calculate the overall cost to deploy and operate a backhaul segment in a small (i.e., $2 \times 2 \mathrm{~km}$ ) dense urban area of an average European city with population density of 3000 users $/ \mathrm{km}$ [12], and with 400 multistory buildings (i.e., 5 floors per building and 2 apartment per floor). Two options are assumed for the wireless deployment: homogeneous (i.e., using macro BSs only), and heterogeneous (i.e., macro BSs are serving outdoor users while small cells are deployed indoor to provide service inside the buildings). Fig. 2 presents the backhaul architectures considered in the case study: microwave only and fiber to the building (FTTB). For the microwave only case, i.e., Fig. 2(a), PtP microwave links are used to backhaul the data traffic from both the macro BS and the small cells. At each building, a switch gathers the data traffic from all its indoor small cells and sends it via a roof top microwave antenna to the closest hub in the area, co-located with one of the macro BSs. The hub then sends the aggregated data traffic to the metro/aggregation network via PtP fiber link(s).

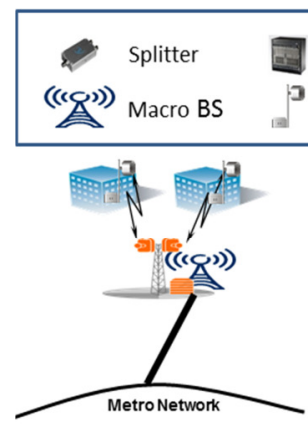

(a)
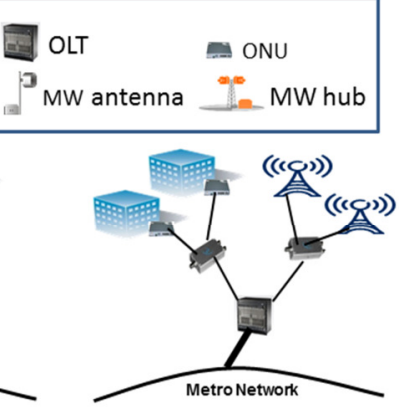

(b)
Fig. 2. Backhaul architectures.

In the scenario with fiber backhauling (Fig. 2(b)), a FTTB architecture is used to collect the data traffic from the indoor users, i.e., the aggregation switch inside each building is now co-located with an ONU connected to an OLT using PON architecture. The macro cells are also backhauled following the same philosophy (i.e., one ONU per macro site, each one connected to OLT in the central office. The central office in turn sends the aggregated traffic to the metro network via PtP fiber link(s). This fiber link is the same in both scenarios, and hence is not considered in the TCO comparison. Since the infrastructure cost is known to be the most expensive component of the TCO in case of fiber networks, two scenarios are considered for the fiber backhaul case. If no fiber infrastructure is available in the area, the MNO will need to deploy (i.e., trench) its own fiber infrastructure (FTTB-Tr). However, if there is already an existing fiber infrastructure available for leasing, the MNO will lease fiber connectivity instead of trenching (FTTB-Le).

It is assumed that the fiber backhaul is based on the 10 GPON (gigabit capable PON) technology providing $80 \mathrm{Mbps}$ per building and $500 \mathrm{Mbps}$ sustainable bandwidth per macro cell. In terms of wireless deployment it is assumed that the homogeneous case comprises a total of 10 macro BSs distributed over the considered area. For the HetNet case the number of macro BSs is 4 , while the number of small cells is 2000 (i.e., 5 per building, one in each floor). In both cases the average capacity value provided over the considered area is the same, i.e., $83 \mathrm{Mbps} / \mathrm{km}^{2}$ [12]. Table I summarizes the cost parameters used to calculate backhaul TCO. The fault management cost is calculated based on the values in [13].

TABLE I. INPUT VALUES USED FOR COST CALCULATION [5][13][14]

\begin{tabular}{|c|c|}
\hline Component/Parameter & Price (Euro) \\
\hline Technician salary (hour) & 52 \\
\hline Energy cost $(\mathrm{kWh})$ & 0,1 \\
\hline Indoor yearly rental fee $\left(\mathrm{m}^{2}\right)$ & 220 \\
\hline Outdoor yearly rental fee $\left(\mathrm{m}^{2}\right)$ & 180 \\
\hline Small/Large microwave antenna & $200 / 2000$ \\
\hline G-Ethernet switch & 1800 \\
\hline Microwave hub + installation & 50000 \\
\hline Ethernet switch & 100 \\
\hline Yearly spectrum leasing per link & 150 \\
\hline GPON/10GPON OLT & $640 / 1750$ \\
\hline GPON/10GPON ONU & $50 / 105$ \\
\hline Power splitter $(1: 16 / 1: 32)$ & $170 / 340$ \\
\hline Fiber $(\mathrm{km})$ & 80 \\
\hline Trenching $(\mathrm{km})$ & 45000 \\
\hline Leasing upfront fee $(\mathrm{km})$ & 800 \\
\hline Yearly fiber leasing fee $(\mathrm{km})$ & 200 \\
\hline
\end{tabular}

Fig. 3 presents the OPEX and CAPEX values for the considered backhaul options over a 20 -year time period. It is evident that backhaul is more expensive in the presence of a HetNet deployment. This confirms the claim made earlier in the paper that it is important to carefully choose the right backhaul architecture and technology in order to minimize the impact on the cost efficiency of a HetNet deployment. In terms of fiber-based solutions, leasing is always the most cost efficient option. But cost is not the only benefit in this case, i.e., with a leased fiber infrastructure the fast deployment is also possible. If a MNO does not have the possibility to lease fibers, microwave is a better option to backhaul macro BS in case of homogeneous deployments. However the power consumption of microwave backhauling is always higher than the one based on fiber technologies (Fig. 4). Fig. 3 shows also that in case of a HetNet deployment, with a large number of small cells, microwave is the most expensive backhaul 
technology. This is due to the component cost and the power consumed by the microwave links, which increase almost linearly with the number of small cells. Therefore, in areas with a high density of small cells, fiber-based backhauling is more cost-efficient, even if an operator needs to deploy its own infrastructure.

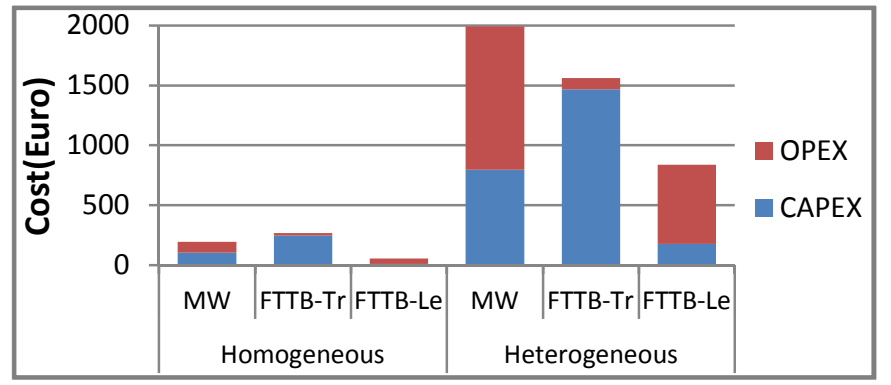

Fig. 3. TCO of mobile backhaul over 20 years.

Fig. 4 presents the cost breakdown to assess the impact of each cost element of the TCO described in Section III, for the three backhaul options considered in the case study. The cost elements are related to: fault management (FM), floor space (FloorSp), spectrum and fiber leasing (Leas), maintenance, energy, infrastructure, and component cost. The presented breakdown can help operators to identify the most expensive parts of the backhaul TCO, which is essential to improve the cost efficiency of the backhaul network. From the figure it becomes evident that each cost item has a different impact on the TCO depending on the deployed technology. For example, FM cost is higher than the energy cost when the operator owns the fiber infrastructure, while it becomes negligible for the other two alternatives. In case of microwave-based backhaul, the rental fee for placing the microwave antennas and hubs is a considerable part of the TCO. This shows the importance of a proper planning and site acquisition strategies in case of microwave backhaul, in contrast to the FTTB-based cases.

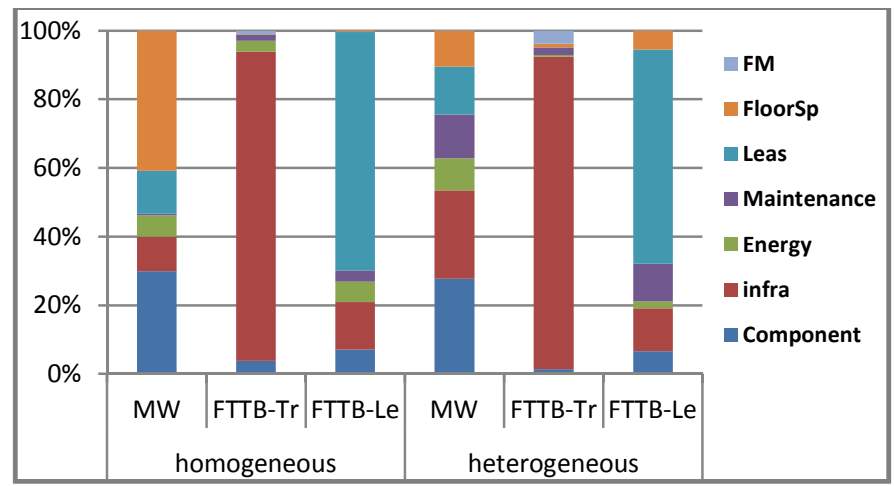

Fig. 4. TCO breakdown.

\section{CONCLUSIONS}

This paper presents a comprehensive model to estimate the total cost of ownership (TCO) of a backhaul network segment. The focus of the paper is on microwave and fiber-based backhaul solutions, which can support high capacity wireless access networks. A case study where the proposed model is applied compares the TCO of backhauling heterogeneous and homogeneous mobile access networks. The results show a considerable increase in the backhaul TCO in the case of a heterogeneous deployment compared to the conventional homogeneous scenario. Based on the presented results, fiber is the most promising technology to provide a high capacity backhaul for the heterogeneous wireless deployments. The cheapest alternative is to lease fiber connectivity when possible. Microwave links on the other hand are suitable to backhaul macro BSs when a fiber infrastructure is not already available. The results also highlight the importance of selecting the right backhaul technology in order to not limit the benefits brought by the heterogeneous wireless deployments. Moreover, a detailed and complete backhaul TCO breakdown allows operators to have a better understanding of their backhaul TCO dynamics thus allowing minimization of CAPEX and OPEX during network deployment and operation. As future work we plan to perform a sensitivity analysis to assess the impact of variances of the input parameters used in the case study.

\section{ACKNOWLEDGMENT}

The authors would like to acknowledge Fabricio Farias for the fruitful technical discussions. The research work was founded by "GreenHaul" a Wireless@KTH seed project and by the European Community's Seventh Framework Program (FP7/2007-2013) under grant agreement $n^{\circ} 318137$ (ICT-DISCUS).

\section{REFERENCES}

[1] S. Tombaz, Z. Zheng, J, Zander, "Energy efficiency assessment of wireless access networks utilizing indoor base stations", IEEE PIMRC 2013.

[2] J. Markendahl, Ö. Mäkitalo, "A comparative study of deployment options, capacity and cost structure for macrocellular and femtocell networks," IEEE PIMRC, 2010.

[3] Vodafone group technology update 2008, http://www.vodafone.com/content/index/investors/reports/company_pres entations.html.

[4] White paper, "How to meet your backhaul capacity needs while maximizing revenue",

http://www.skyfiber.com/assets/docs/pdf/technologyandresouces/WP_S kyFiber_Mobile_Backhaul.pdf, 2013.

[5] A. Ahmed, J. Markendahl, C. Cavdar, A. Ghanbari, "Study on the effects of backhual solutions on indoor mobile deployment macrocell vs. femtocell”, IEEE PIMRC 2013.

[6] Z. Frias, J. Peres, "Techno-economic analysis of femtocell deployment in long-term evolution networks", EURASIP Journal on Wireless Communications and Networking, vol. 2012, no. 1, pp. 1-15, 2012.

[7] W. S. Soh, Z. Antoniou, H.S. Kim, "Improving restorability in radio access networks", IEEE GLOBECOM 2003.

[8] F. C. Kuo, F.A. Zdarsky, J. Lessmann, S. Schmid, "Cost efficient wireless mobile backhaul topologies: an analytical study", IEEE GLOBECOM 2010.

[9] M. Paolini, "An analysis of the total cost of ownership of point-to-point, point-to-multipoint, and fibre options", White paper on crucial economics for mobile data backhaul, 2011.

[10] P. Monti, S. Tombaz, L. Wosinska, J. Zander, "Mobile backhaul in heterogeneous network deployments: technology options and power consumption", IEEE ICTON 2012.

[11] Heterogeneous networks, TIA 2012|@Ericsson http://tia2012.org/sites/default/files/pages/Coyne\%20Michael.pdf

[12] EARTH D2.3, "Energy efficiency analysis of the reference systems, areas of improvements and target breakdown".

[13] M. Mahloo, C.M. Machuca, J. Chen, L. Wosinska, "Protection cost evaluation of WDM-based next generation optical access networks", Optical switching and networking journal,vol. 10, no. 1, pp. 89-99, 2013.

[14] Porta Optica D3.2v3: Economic analysis, dark fibre usage cost model and model of operations, http://www.porta-optica.org/publications/POSD3.2_Economical_analysis.pdf. 